\title{
New Methods for Analyzing Complex Biomedical Systems and Signals
}

\author{
Irini Reljin $(\mathbb{D}){ }^{1}$ Zoran Obradović $\mathbb{D}^{\mathbb{D}},{ }^{2}$ Mirjana B. Popović, ${ }^{1,3}$ and Valeri Mladenov $\mathbb{D}^{4}$ \\ ${ }^{1}$ School of Electrical Engineering, University of Belgrade, 11000 Belgrade, Serbia \\ ${ }^{2}$ Center for Data Analytics and Biomedical Informatics, Temple University, Philadelphia, PA 19122, USA \\ ${ }^{3}$ Institute for Medical Research, University of Belgrade, 11000 Belgrade, Serbia \\ ${ }^{4}$ Department of Theoretical Electrical Engineering, Technical University of Sofia, 1000 Sofia, Bulgaria \\ Correspondence should be addressed to Irini Reljin; irinitms@gmail.com
}

Received 13 March 2018; Accepted 13 March 2018; Published 24 May 2018

Copyright (C) 2018 Irini Reljin et al. This is an open access article distributed under the Creative Commons Attribution License, which permits unrestricted use, distribution, and reproduction in any medium, provided the original work is properly cited.

In recent years, there has been significant progress in the applications of sensing devices in acquiring biomedical signals. Availability of big and/or high dimensional data obtained by new instruments inspired the development of novel information processing methods aimed at significantly improving the understanding of subtle and complex mechanisms of biomedical processes. Such data have proved useful in diagnosis, prediction of health state, survival prognosis, and therapy optimization. New sensing devices generate precise signals, large-scale biomedical data which has opened challenging problems for managing, processing, storing, retrieving, transferring, and interpreting such data. Therefore, we have invited the researches to contribute their research on new algorithms and tools for modeling, processing, and analyzing complex biomedical signals. After rigorous review, this special issue accepted eleven articles on this topic which are summarized here.

In the paper "High-Density Lipoproteins-Associated Proteins and Subspecies Related to Arterial Stiffness in Young Adults with Type 2 Diabetes Mellitus," X. Zhu et al. analyzed the correlation of phospholipid-associated protein with a pulse wave velocity (PWV) in order to find which protein is related to arterial stiffness in persons with type 2 diabetes mellitus (T2D). Five adolescents with T2D and six lean controls with no evidence of chronic disease were under consideration. By integrating patient clinical data, biological experiments, and bioinformatics analysis to reveal arterial stiffness-related high-density lipoproteins and subspecies, the authors found, among 68 detected phospholipid-associated proteins, seven as negatively correlated with PWV, indicating they may be atheroprotective, and nine proteins as positively correlated with $\mathrm{PWV}$, suggesting they may be related to arterial stiffness.

Pathological tremor is a complex movement disorder of different causes, affecting about $5 \%$ of the population over the age of 65. The quantification and discrimination of Parkinsonian disease (PD) and essential tremor (ET) have significant impact on both patient treatment and the development of new interventions in clinical practice. In the paper "New Perspectives for Computer-Aided Discrimination of Parkinson's Disease and Essential Tremor," P. P. Bržan et al. proposed novel metrics to quantify and discriminate PD and ET tremor. These metrics, as well as other known tremor-related movement metrics, were tested over $27 \mathrm{PD}$ and 27 ET patients. The discriminatory power of all of these metrics was evaluated based on the receiver operating characteristics, decision tree modeling, and binary logistic regression. After testing the discriminative power of individual metrics, it was found that the new metrics outperformed alternative methods.

Auscultation is widely used for evaluation of cardiac function. Such analysis mostly depends on the individual skills of the physician, and therefore there is a growing demand for automatic heart sound interpretation methods. The initial step in heart sound analysis is to identify primary heart sounds, S1 and S2. Proper identification of these sounds is of key importance for identifying other components of 
heart signal, such as extra sounds and murmurs. In the paper "Identification of S1 and S2 Heart Sound Patterns Based on Fractal Theory and Shape Context," A. Gavrovska et al. proposed a new method for identifying and differentiating primary heart sounds S1 and S2, without the electrocardiogram reference. The new method is based on adaptive filtering and a modified blanket approach, which describe the shape of the heart signal. After determining proposed characteristic descriptors, the SVM-based classification and the fivefold cross-validation have been performed. Nine hundred sound sequences collected from the University Children's Hospital in Belgrade, Serbia, were analyzed. The evaluation results are obtained by using the receiver operating characteristic (ROC) curve. Calculated AUC (area under the curve) and the classification accuracy and F-measure were used as performance measures. Results are compared with other known shape related methods. The proposed method, using only three descriptors, is of high accuracy (higher than 95\%) and outperforms other methods by at least $6 \%$.

In the paper "Follow-Up and Risk Assessment in Patients with Myocardial Infarction Using Artificial Neural Networks," T. Gligorijević et al. described a novel method for identifying individuals with high risk of death after acute myocardial infarction by using artificial neural networks (ANNs). Training data for ANN was 1,705 patients admitted between 2003 and 2013 to Coronary Care Unit of Clinical Hospital Center Bežanijska Kosa, Belgrade, Serbia, who underwent 24-hour ECG monitoring, short ECG analysis, noninvasive beat-to-beat heart rate variability, and baroreflex sensitivity. The proposed neural network classifier showed good performance for survival prediction: $88 \%$ accuracy, $81 \%$ sensitivity, 93\% specificity, $0.85 \mathrm{~F}$-measure, and area under the curve value of 0.77 . These findings support the theory that patients with high sympathetic activity (reduced baroreflex sensitivity) have an increased risk of mortality independent of other risk factors and that artificial neural networks can indicate higher risk individuals.

The approximate entropy (ApEn) is one of the most used nonlinear techniques for quantifying the complexity and unpredictability of time series. The ApEn is approved as a supporting tool in preclinical and clinical studies, with the most prominent applications in cardiovascular studies. One of the very first ApEn modifications is the CrossApEn (XApEn) that estimates mutual predictability of two simultaneously recorded time series. In view of ApEn as a complexity measure of solitary signal, XApEn should have become an equivalent tool for paired physiological processes. In the paper "On Consistency of Cross-Approximate Entropy in Cardiovascular and Artificial Environments," T. Skoric et al. studied the problems that preclude wider XApEn implementation and proposed methods for their adjustment. Their study used three types of data: artificial (for testing the stability of estimated values), animal (for method development and for repeated testing), and human (to validate the derived methods in typical real applications where the records are short). The new approach is verified by using cardiovascular signals recorded from rats (long signals) and healthy volunteers (short clinical signals), proposing a change of traditional parameter guidelines.
In the paper "Sparse Learning of the Disease Severity Score for High-Dimensional Data," I. Stojkovic and Z. Obradovic proposed a novel approach to the problem of learning disease severity scores in presence of irrelevant or high-dimensional measurements. The proposed formulation of sparse severity score learning forces weights of most of the features to be exactly zero, therefore effectively performing feature selection by learning the sparse linear scoring function. This novel severity score objective function is convex and nonsmooth and it precludes the direct use of convenient optimization tools like gradient-based methods. They defined the severity score as a linear combination of intensities of the most relevant features. The proposed approach might be used as an effective and reliable tool for both scoring function learning and biomarker discovery, as demonstrated by identifying a stable set of genes related to influenza symptoms' severity, which are enriched in immune-related processes.

Modeling and representing biological processes are a challenging problem for biochemical researchers. Computational systems biology is becoming a fundamental tool of life-science research, which aims at developing models representing biological phenomena and reliable computational techniques for their simulation. In the paper "HSimulator: Hybrid Stochastic/Deterministic Simulation of Biochemical Reaction Networks," L. Marchetti et al. considered this problem and described the simulator for mass-action reaction. They combined several strategies including exact stochastic simulation, deterministic simulation, and hybrid simulation. For deterministic and hybrid simulations, the simulator automatically translates the mass-action reaction network into a set of ordinary differential equations suitable for further resolving. Their simulator provides a suite of state-of-the-art simulation algorithms including the exact algorithm, RSSA (rejection-based stochastic simulation algorithm), and the first publicly available implementation of its hybrid version, HRSSA. The benchmarks in the paper show that their simulator implementation is often faster than the state-ofthe-art COPASI (complex pathway simulator) simulator.

In the paper "Interpolative Boolean Networks," V. Dobrić et al. proposed a generalized Boolean network adaptive with respect to the nature of input variables which offers greater descriptive power as compared with traditional models. Instead of classical Boolean networks, which are binary, by introducing the gradation in this model more descriptive power can be provided. This approach, called by authors the interpolative Boolean networks, is based on interpolative Boolean algebra: the $[0,1]$-valued realization of Boolean algebra. Interpolative Boolean networks can be used to predict behavior of complex systems. For the purpose of their practical application, a simple software tool is developed. When applied on two examples from the literature and compared against other real-valued logical approaches, the proposed interpolative Boolean networks provided a superior descriptive power.

In the paper "Monitoring Effective Connectivity in the Preterm Brain: A Graph Approach to Study Maturation,” M. Lavanga et al. investigated effective electroencephalography (EEG) based brain connectivity in premature infants, whose postmenstrual age ranged from 27 to 42 weeks. Results 
showed that the EEG-graphs changed with age in terms of topology. In particular, the clustering coefficient and the spectral radius decreased with maturation, while the path length increased. Results showed that the EEG-connectivity, assessed using graph theory indices, moved from a smallworld network to a random one, since the clustering coefficient increases and the path length decreases. Their results showed that it is possible to predict the age of the infant with a root mean-squared error equal to 2.11 weeks. These results are consistent with the ones reported in the literature for age prediction in preterm babies.

Over the last decade, various methods were proposed for characterizing human emotions from electroencephalogram (EEG). In the paper "Factor Analysis for Finding Invariant Neural Descriptors of Human Emotions," V. Pereira et al. proposed a new spatial-temporal feature set with clear physical ground over selected EEG electrodes and the factor analysis (FA) as an alternative method to extract the most important components of the feature set. In contrast to the widely used principal component analysis, FA preserves the relation between the factors and the feature set and therefore the conclusions are more easily interpreted by psychologists and neurophysiologists. The combination of the proposed features and FA resulted in finding the most representative neural descriptors of the emotion valence, invariant with respect to trials and subjects. The authors provided evidence that the factor analysis of event-related potentials is a promising approach to extract statistical underlying correlations of the brain activity among subjects and therefore decode human emotional states. By extracting temporal and spatial EEG features, they showed that it is possible to build Brain Computer Interface for decoding human emotions across various subjects.

In the paper "Vector Autoregressive Hierarchical Hidden Markov Models for Extracting Finger Movements Using Multichannel Surface EMG Signals," N. Malešević et al. presented a novel computational technique intended for the robust and adaptable control of a multifunctional prosthetic hand using multichannel surface electromyography (EMG). Their study indicates that using mean absolute value feature coupled with hierarchical hidden semi-Markov models algorithm leads to movement decoding accuracy higher than other combinations of features and classifiers. This combination also guaranties the shortest motion selection and motion completion times, influencing a response of a prosthetic hand to a user intent. Their algorithm is characterized by low computational complexity for the execution, easy expansion, and noise resiliency.

We believe this special issue is useful for investigators who work with complex biomedical signals. We hope that the papers from this issue will initiate further interest in analyzing complex biomedical systems.

\section{Acknowledgments}

The guest editorial team thanks the authors who submitted their papers to this special issue. Also, our sincere thanks go to reviewers for their dedicated efforts in evaluating the submitted papers and providing helpful comments. Finally, we thank the journal's Editorial Board for their help and assistance.

\author{
Irini Reljin \\ Zoran Obradović \\ Mirjana B. Popović \\ Valeri Mladenov
}




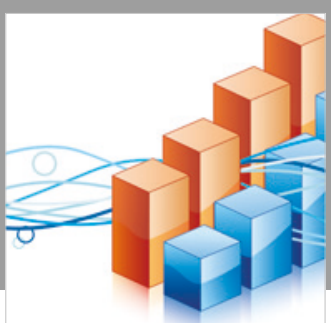

Advances in

Operations Research

\section{-n-m}

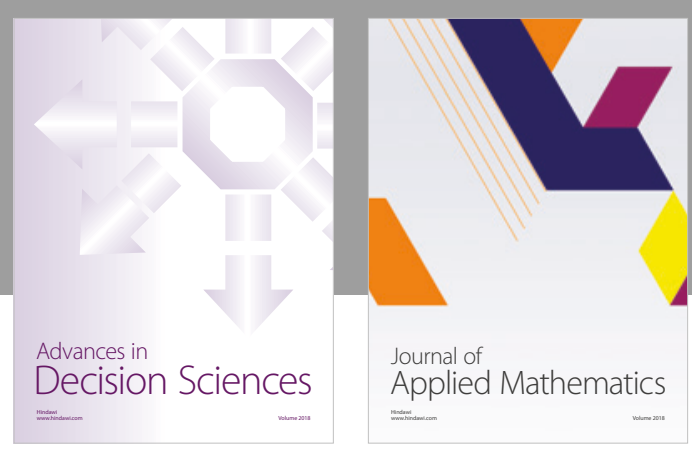

Journal of

Applied Mathematics
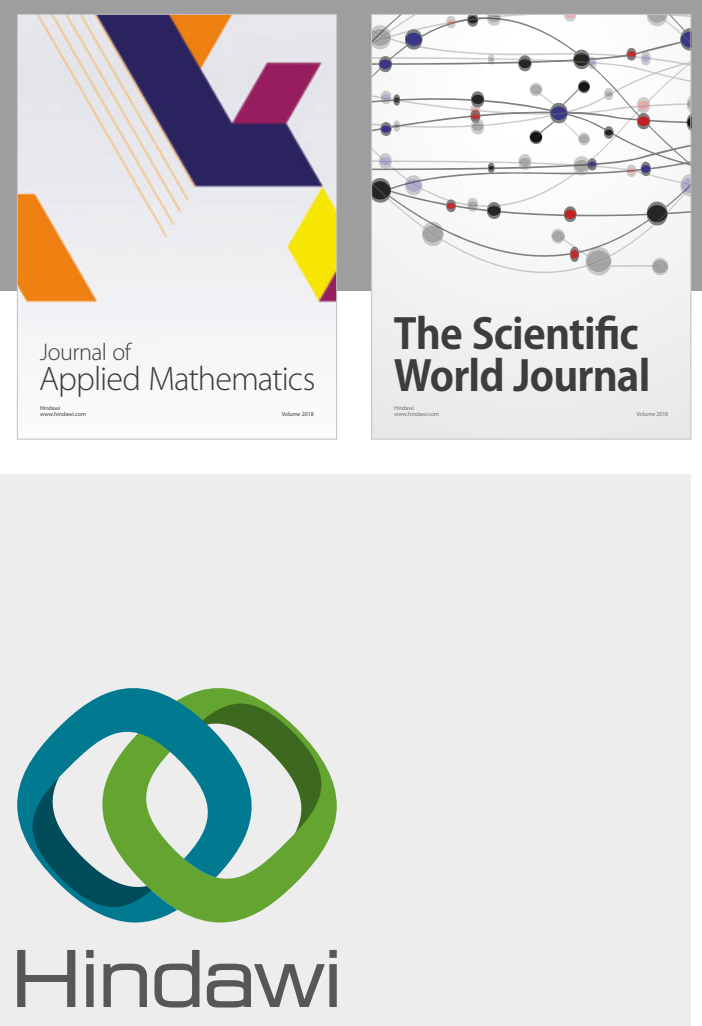

Submit your manuscripts at

www.hindawi.com

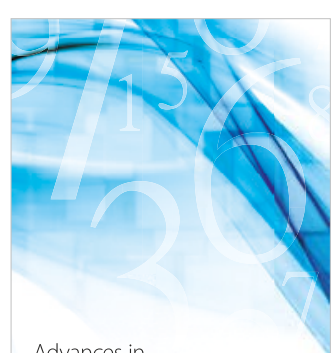

Advances in
Numerical Analysis
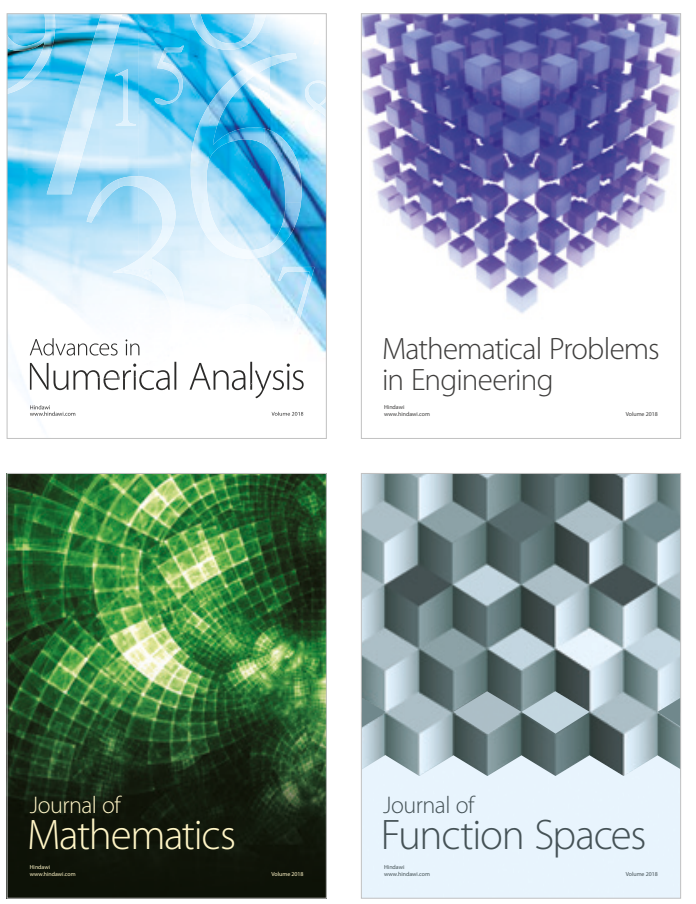

Mathematical Problems in Engineering

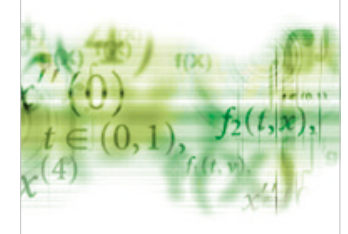

International Journal of

Differential Equations

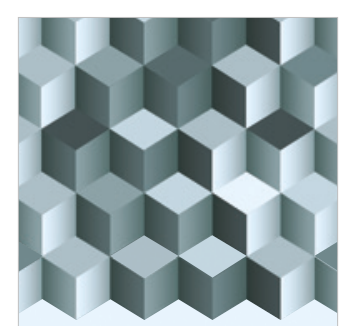

Journal of

Function Spaces
The Scientific

World Journal

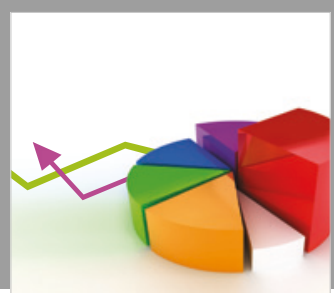

Journal of

Probability and Statistics
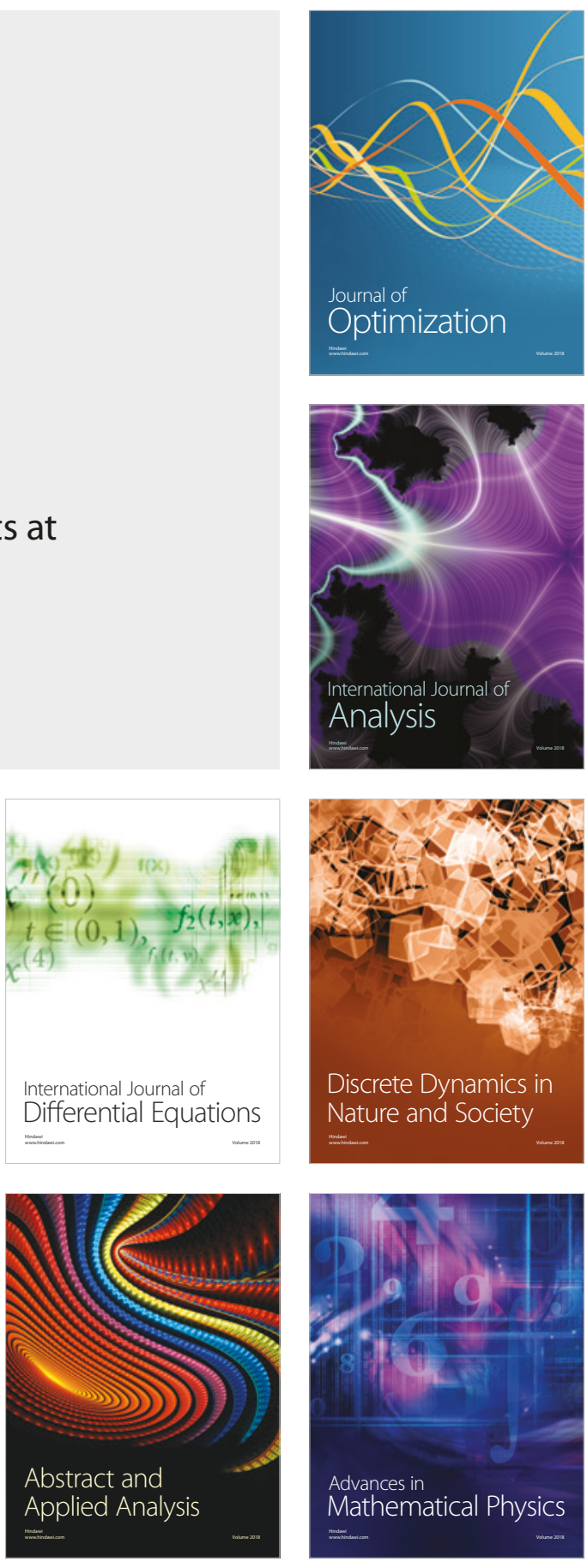